\title{
Association of Oral Health Literacy Level and Periodontal Health Status: A Contributory Step Towards Prevention of Periodontal Diseases
}

\author{
Wardah Ahmed ${ }^{1}$ \\ Mehmil Aslam² \\ Syed Imran Hassan ${ }^{3}$ \\ Khalil Ahmed $^{4}$ \\ Syeda Farhat Bukhari ${ }^{5}$ \\ Permanand Mahi ${ }^{6}$ \\ Ayesha Kaleem ${ }^{7}$ \\ Fatima Zafar ${ }^{8}$
}

\author{
BDS, MSPH, PhD \\ BDS, MCPS \\ BDS, MCPS \\ BDS, MSc \\ BDS, MSc \\ BDS, MSc \\ BDS \\ BDS
}

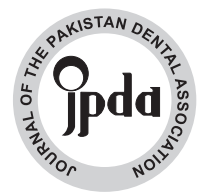

OBJECTIVE: To Evaluate the level of oral health literacy (OHL) among new patients seeking care at the private teaching dental hospital. METHODOLOGY: A cross-sectional study conducted at Department of Oral Diagnosis \& Periodontology at Private Teaching Dental Hospital. Adults (>18years) was recruited in the study using non-probability convenient sampling technique. Participants verbally consented to participate, then interview based validated questionnaire was administered. Data collected from April 2019 to August 2019 . The study was approved by Scientific Review Committee of Private Teaching Dental Hospital. OHL was measured by REALD-20. Clinical assessment was performed using periodontal index. Two investigators and 2 house surgeons were trained for increasing inter-examiner reliability. Demographic variables were examined as exploratory covariates. Statistical analysis was performed using SPSS 21.Fisher's exact test used to determine bivariate association and Multivariate regression analysis showed the relationship.

RESULTS: The total sample size was $n=236$ out of which $100(42.6 \%)$ were male and $136(57.6 \%)$ were female. Mean age of the sample was 36 years $( \pm 13.6)$ and majority $150(64 \%)$ had 12 years of education completed. Smokers were $50(21 \%)$ were smokers. Respondents $173(73.3 \%)$ brush at least once in a day and 93\% used toothpaste as dentifrice. Respondents belong to low-OHL had moderate 84 (36\%) and severe periodontal $72(31 \%)$ diseases. The mean Periodontal Index Score: $2 \pm 0.54$. OHL was significantly associated with periodontal disease (p 0.01). Smokers showing significant association ( $\mathrm{p}$ 0.04) with periodontal disease. Multivariate regression analysis revealed that for one- point decrease in OHL score, the occurrence of periodontal disease reduced by $25 \%$ (Adjusted OR=0.75).

CONCLUSION: This has been concluded that oral health literacy associated with periodontal health status. Understanding of periodontal health knowledge and its implication is vital for prevention of periodontal diseases. Future intervention researches are required to develop etiological relationship between oral health literacy and periodontal health status.

KEYWORDS: Periodontal disease, dental oral health, smokes adults

HOW TO CITE: Ahmed W, Aslam M, Hassan SI, Ahmed K, Bukhari SF, Mahi P, Kaleem A, Zafar F . association of oral health literacy level and periodontal health status: a contributory step towards prevention of periodontal diseases. J Pak Dent Assoc 2021;30(3):164-169. DOI: https://doi.org/10.25301/JPDA.303.164

Received: 07 September 2020, Accepted: 06 May 2021

\section{INTRODUCTION}

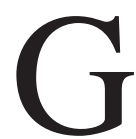

enerally Oral Health Literacy (OHL) conceptualized as the degree to which individuals have the capacity to obtain, process, and understand

1. Assistant Professor, Department of Community Dentistry, Hamdard College of Medicine and Dentistry.

2. Assistant Professor, Department Oral Medicine, Hamdard College of Medicine and Dentistry

3. Professor, Department of Periodontology, Hamdard College of Medicine and Dentistry.

4. Assistant Professor, Department of Orthodontic, Bolan University of Medical Health Sciences, Quetta.

5. Assistant Professor, Department of Dental Materials, Hamdard College of Medicine and Dentistry.

6. Assistant Professor, Department of Operative Dentistry, Bhitai Dental and Medical College, MirpurKhas.

7. Lecturer, Department of Periodontology, Hamdard College of Medicine and Dentistry

8. House Surgeon, Department of Periodontology, Hamdard College of Medicine and Dentistry.

Corresponding author: "Dr. Wardah Ahmed" < wardahahmed83@ hotmail.com > basic oral health information and services needed to make appropriate health decisions. ${ }^{1}$ Low health literacy identified as the main hindrance in health care utilization and could be a key factor in negative health outcomes of particular interest and concern for vulnerable population. ${ }^{1,3,4}$ Unable to self-rating of health, less understanding of prevention and self-care instructions, less compliance to adherence to medicinal and health instructions, compromised selfmanagement skills, high burden of mortality and morbidity hazards directly linked to low health literacy that consequently increase in healthcare costs. ${ }^{5-7}$ Previous studies have shown that marginalized populations for low health literacy effected from inequalities in health status, they are more prone to diseases like cardiovascular disease, diabetes 
mellitus, obesity, HIV, malignancy, lead poisoning and low birth weight. ${ }^{8-10} \mathrm{OHL}$ seen as a major contributing risk factor of an individual oral health behavior and oral health status. ${ }^{11-}$ ${ }_{13}$ Periodontal disease is a term "which includes all pathological conditions of the periodontium". Periodontal problem are true infection of oral cavity. Periodontal diseases defined as "infectious progressions that require bacterial presence and a host response and are further affected and modified by other local, environmental and genetic factors". Periodontal infection is instigated by specific invasive oral pathogens that colonize dental plaque biofilms on dental enamel in the absence of oral hygiene maintenance. ${ }^{14}$ Thus, it has been evidently proved that oral hygiene has direct relationship with periodontal diseases. Periodontal diseases are preventable and maintained with patient compliance. OHL linked with deeper understanding of oral care information and its application for observance to the management of periodontal disease. ${ }^{15}$ Nevertheless, patient strict compliance may help in successful treatment of the causes of all stages of periodontal diseases. ${ }^{16}$ Additionally, oral health status correlated with quality of life that ultimately associated with periodontal diseases. ${ }^{17,18} \mathrm{OHL}$-instruments comprised of contemporary measures of oral health literacy expertise. Broadly, used oral health literacy measurement tools derived from Rapid Estimate of Adult Literacy in Dentistry

(REALD-99), Rapid Estimate of Adult Literacy in Medicine and Dentistry (REALM-D) administered for word recognition and Test of Functional Health Literacy in Dentistry (TOFHLiD) used to assess reading comprehension and numeracy. In literature, conceptual knowledge assessed form comprehensive oral health knowledge (COHK) tool and general oral health knowledge tool. Oral health knowledge (OHK) considered as independent component of oral health literacy effecting the oral health outcomes. Conceptual knowledge helps in understanding and decision making component of oral health literacy. These tools comprise of extensive items and questions related to oral health. ${ }^{19-23}$

Periodontal diseases are among the most widely prevalent disease of oral cavity worldwide. ${ }^{4,24,25}$ In Pakistan overall prevalence of periodontal diseases is $80 \%$ as reported in situation analysis of Pakistan. ${ }^{26}$

The rationale of conducting this study that general literacy level in the urban Karachi is reportedly $70 \% .{ }^{28}$ Basic Oral health knowledge of regarding oral hygiene is almost universal that covers knowledge about usage of toothbrush and toothpaste. ${ }^{26}$ However, the occurrence of periodontal diseases is high. In previous study it was found that almost $70 \%$ of adult population have limited oral health literacy.$^{29}$ Prevention of periodontal diseases and promotion of healthier oral care requires understanding of consequences of periodontal disease. Nevertheless, oral health literacy augment people's ability to read and write. The knowledge gained from understanding periodontal disease and how best to reach individuals with low OHL will help in providing optimal periodontal care, education, and support. ${ }^{15}$

In the context of Pakistan and to the basis existing scarce literature, this kind of study conducted for the first time. This study assess the association between oral health literacy and periodontal health status.

The specific objectives of this study:

1. Estimate the level of Oral Health Literacy (OHL) among new patients utilizing care at the private teaching dental hospital

2. Assess association of Oral health Literacy (OHL)with periodontal health status

3. Assess association of Oral health knowledge (OHK) with periodontal health status

\section{METHODOLOGY}

\section{Sample and Data Collection}

Newly visited adult patients (>18years of age) to Department of Oral Diagnosis \& Periodontology at a private teaching dental hospital were recruited in the study using non-probability convenient sampling technique. It was cross sectional study. Medical records number was used for marking unique identity of respondent for the study. Patient with other morbidity such as acute dental pain and oral cancers was not included in the study. If they verbally consented to participate, then interview based validated structured questionnaire was administered. Sample size was calculated using software "OpenEpi". Prevalence of low oral health literacy $(31 \%)$ in previous study was taken as reference to estimate sample size. ${ }^{11}$ The calculated sample size turned out to be $n=236$ at $95 \%$ confidence level at power 0.05. Data collected from September 2018 to April 2019. Principal investigator, 2 co-authors and 2 house surgeons trained

and calibrated in a single training session for increasing inter-examiner reliability. This study was approved by Scientific Review Committee of the private teaching dental hospital.

\section{Data Collection Tool}

The tool used for data collection had 4 sections. Section 1: Socio-demographic characteristics including age, gender and education. In addition, the oral health behavior section assessed through six items on dental hygiene and habits. 
Section 2: Oral health literacy (OHL) measurement tool adapted from Rapid Estimate of Adult Literacy in Dentistry (REALD-20).The REALD involved "word recognition test" that assess respondents' capacity to read dental words from a preformed list and interpreted as grade-range assessments of reading capability. REALD-20 administered to evaluated OHL by using flash cards. Dentistry related 20 words written on flash cards from less difficult to more difficult word consulted and piloted with periodontology experts. Cronbach's $=0.87$ showed instrument validation. Each respondent was asked to read loudly the words written on flash cards. If there was any difficulty in reading and recognition by the respondents, interviewer asked to skip the word. Collectively, score (0-20) was enumerated, 0 marked as lowest and 20 as highest. Distribution of respondents done, based on these OHL- Categories (i) low $(\leq 14)$, (ii) moderate (15 to 17$)$ and (iii) high $(\geq 18)$.

Section 3: Comprehensive oral health knowledge (CMOHK) and Oral health knowledge (OHK) comprised of 9 questions administered to knowledge specific to periodontal disease. Although considering independent component, oral health knowledge helps in understanding and decision making for the better outcomes. Single correct answer of true/false response item marked as score 1.

Section 4: Periodontal health status assessed through clinical assessment. Periodontal diseases was measured using periodontal index. Widely used periodontal disease case definition in accordance with CDC used in this study: Severe Periodontitis: $\geq 2$ interproximal sites with CAL $\geq 6$ $\mathrm{mm}$ (on more than one tooth) and $\geq 1$ interproximal site with PD $\geq 5 \mathrm{~mm}$;

Moderate Periodontitis: $\geq 2$ interproximal sites with CAL $\geq 4 \mathrm{~mm}$ (on more than one tooth) or $\geq 2$ interproximal sites with PD $\geq 5 \mathrm{~mm}$ (on more than one tooth);

Healthy or Mild Periodontitis: neither "moderate" nor "severe" periodontitis". ${ }^{11}$

\section{Data Analysis}

Outcome variable was periodontal health status. The independent variable was REALD-20 score. Other covariates included gender; education (college, graduate and post graduate); smoking status whether present or previous smokers; OHK and CMOHK score. Statistical analysis done by using SPSS 21. Descriptive statistics reported in percentages for categorical variables (Education, Oral health Literacy level, distribution of respondent according to periodontal health status)and mean and standard deviation for numeric variables (age). Fisher's exact test statistics was used to determine association. Multivariate regression analysis was used to see the relationship between the significant independent variables and outcome.

\section{RESULTS}

\section{Socio-Demographic Characteristics}

The total sample size was $n=236$ out of which 100 $(42.6 \%)$ were male and $136(57.6 \%)$ were female. Respondents sample mean age was 36 years $( \pm 13.6)$, majority $150(64 \%)$ had 12 years of education completed while 72 $(30 \%)$ completed their graduation.

\section{Oral Health Behavior}

In the study sample, 50 (21\%) were smokers, covered current and former smokers and all were male. $\mathrm{Pan} / \mathrm{chhalia/tobbaco} \mathrm{chewing} \mathrm{and} \mathrm{other} \mathrm{addictive} \mathrm{items} \mathrm{were}$ prevalent in $15 \%$ respondents. Majority of the respondents $173(73.3 \%)$ brush at least once in a day and $93 \%$ used toothpaste as dentifrice. Flossing and mouthwash rinses was not adopted by $195(83 \%)$ and $189(80 \%)$ respondents respectively.

\section{Oral Health Knowledge (OHK) and Comprehensive} Measure of Oral Health Knowledge (CMOHK)

Distribution of respondents in questions related to OHK and CMOHK tabulated in table -1. Majority of the respondents had incorrect knowledge about periodontal diseases. Similarly, mostly responses were incorrect regarding comprehensive knowledge.

Table 1: Periodontal Health Status by Oral Health Literacy Level (OHL) and Oral Health knowledge (OHK)

\begin{tabular}{|ll|c|c|c|c|c|}
\hline & & \multicolumn{3}{|c|}{ Periodontal Health Status } & Total & p-value* \\
\hline \multirow{5}{*}{ OHL-Level } & $\begin{array}{c}\text { Mild } \\
\text { PD }\end{array}$ & Moderate PD & Severe PD & & \\
& Low & 1 & $\underline{84}$ & $\underline{72}$ & 157 & \\
\cline { 2 - 8 } & Moderate & 0 & 25 & 7 & 32 & 0.001 \\
\cline { 2 - 8 } & High & 0 & 36 & 11 & 47 & \\
\hline \multirow{2}{*}{ Total } & $\mathrm{n}$ & 1 & 145 & 90 & 236 & \\
\hline \multirow{2}{*}{ OHK } & Low & 1 & $\underline{111}$ & $\underline{64}$ & 176 & \\
\cline { 2 - 9 } & Moderate & 0 & 34 & 26 & 60 & 0.319 \\
\cline { 2 - 9 } & High & 0 & 0 & 0 & 0 & \\
\hline Total & $\mathrm{n}$ & & 145 & 90 & 236 & \\
\hline
\end{tabular}

*Level of Significance 0.05

* t- test

\#PD-Periodontal Disease

\section{Periodontal Health Status by Oral Health Literacy (OHL) Level}

The proportions of respondents among mild, moderate and severe periodontal disease according to oral health literacy (OHL) level illustrated in figure 1. Respondents belong to low oral health literacy level had moderate 84 $(36 \%)$ and severe periodontal $72(31 \%)$ diseases. The mean Periodontal Index Score: $2 \pm 0.54$. 
Ahmed W/ Aslam M/ Hassan SI/ Ahmed K/

Bukhari SF/ Mahi P/ Kaleem A/ Zafar F

Association of Oral health Literacy with Periodontal Disease

Bivariate analysis showed Oral health literacy was significantly associated with periodontal disease (p 0.001).There was no significant difference found among oral health knowledge regarding their periodontal health status.

Although, $21 \%$ of the respondents were smokers in our study, however, it was significantly associated ( $p$ 0.04) with periodontal disease. Other characteristics such as age and education were not significantly associated.

Multivariate regression analysis revealed that for one-point decrease in OHL score, the occurrence of periodontal disease reduced by $25 \%$ (Adjusted $\mathrm{OR}=0.75$ ). Similarly, respondents who smokes (Adjusted OR $=1.58$ ) were significantly more likely intended to have periodontal disease. This demonstrates that people with higher oral health literacy level tended to achieve better periodontal health status.

\section{DISCUSSION}

Pakistan is a developing country and problems such as increase in population growth, low economic resources and burden of communicable and non-communicable diseases escalating exponentially. Thus, oral diseases grasp comparatively less importance and resources allotment than other diseases with high mortality rate. Little is known about dental utilization or unmet dental need in this population and the existing dental care delivery system has failed to reduce the burden of oral diseases in the country. 27,29

This study aim to estimate the level of oral health literacy among new patients utilizing care at private dental hospital in the department of Oral Diagnosis and Periodontology and to assess its association with periodontal health status on the targeted population. Result findings determined that oral health literacy score reduced by 1 unit, probability of getting severe periodontal disease was increased by 25 percent. Similar to our results other studies reported majority of the respondents scored in low OHL level. ${ }^{28,29}$

In our results education and age were not significantly associated to periodontal diseases. This finding advocates in the favor that years of education have no direct relationship with oral health literacy level of the individual. Thus, even highly educated patients have scarce understanding of periodontal issues contributing in development of the disease. ${ }^{12,13}$

Our analysis revealed that few respondents from sample were habitual smokers, still periodontal diseases (mild, moderate and severe) strongly associated with smoking.
Association of oral health literacy level and periodontal health status
Therefore, emphasize not on just knowledge rather understanding and application of healthy behavior. Assessment of oral health knowledge and comprehensive health knowledge revealed that respondents were aware of conditions that require tertiary care. However, it was found that knowledge regarding initial stage of periodontal disease which can be prevented, were lacking. For example, a question what are the behaviors that may cause periodontal disease? Majority of the respondents incorrectly respondent that improper brushing may cause gum disease. These findings consistent with other studies conducted in developed countries found that low oral health literacy interconnected to less oral health knowledge and increasing risky oral-health behavior. ${ }^{7,24,30}$ It is inevitable for the patient to understand the contributing risk factors and causes of periodontal disease. ${ }^{11}$ Sociocultural and environmental factors play imperative part in oral disease and general health consistent with results demonstrated in several reports. ${ }^{23,30}$ The link between socio economic status of the patient and OHL was also recorded but most of the patients were reluctant to give any information, therefore the relationship between these two cannot be evaluated.

Results of this study strengthen the evidence of fruitful two-way communication between dentist and patient. Despite, a report claimed that due to overburden health providers might not conveniently address the basic health literacy requirements, yet this can be resolved by effective oral health education programs and implication for their patients. ${ }^{12}$ Similar pattern found in recent study in Pakistan. Low oral health literacy could be address by simple measures include observing reading capacity of informed consent, prescription reading, medicines leaflets and other patient to identify patients with low literacy. ${ }^{29}$

The study sample limited to the patients visited to OPD in private teaching dental hospital, who already have some level of dental literacy that might overestimated the OHL-score. Furthermore, it would be useful to enlarge the sample size for increasing generalizability of the results in general population. Future, interventional studies in communities encompassing dental health literacy proven to be effective and have positive impact on oral health status. $^{31}$

\section{CONCLUSION}

This has been concluded that oral health literacy associated with periodontal health status. Understanding of periodontal health knowledge and its implication is vital for prevention of periodontal diseases. Future intervention researches are required to develop etiological relationship between oral health literacy and periodontal health status. 
Ahmed W/ Aslam M/ Hassan SI/ Ahmed K/

Bukhari SF/ Mahi P/ Kaleem A/ Zafar F

\section{CONFLICT OF INTEREST}

None to declare

\section{REFERENCES}

1. Chowdary MS, Sudhir KM, Reddy VC, Kumar K, Srinivasulu G. Oral health literacy and its impact on oral health status among institutionalised elderly population. IOSR J Dent Med Sci. 2015;14:96104

https://doi.org/10.9790/0853-148296104

2. Horowitz AM, Kleinman DV. Oral health literacy: the new imperative to better oral health. Dental Clinics of North America. 2008;52: 333-44.

https://doi.org/10.1016/j.cden.2007.12.001

3. Nutbeam D. Health literacy as a public health goal: a challenge for contemporary health education and communication strategies into the 21 st century. Health promotion international. 2000;15:259-67. https://doi.org/10.1093/heapro/15.3.259

4. Parker EJ, Jamieson LM. Associations between indigenous Australian oral health literacy and self-reported oral health outcomes. BMC Oral health. 2010;10:1-8.

https://doi.org/10.1186/1472-6831-10-3

5. Toçi E. Health literacy in the Western Balkans. 2015.

6. Nutbeam D. The evolving concept of health literacy. Social science \& medicine. 2008;67:2072-078.

https://doi.org/10.1016/j.socscimed.2008.09.050

7. Griffey RT, Kennedy SK, McGownan L, Goodman M, Kaphingst KA. Is low health literacy associated with increased emergency department utilization and recidivism? Academic Emergency Medicine. 2014;21:1109-15.

https://doi.org/10.1111/acem.12476

8. Tutu RA, Busingye JD. Health Literacy of Migrants: Environmental Risks to Health. Migration, Social Capital, and Health: Springer; 2020. p. 71-96.

https://doi.org/10.1007/978-3-030-24693-8_5

9. Williams MV, Baker DW, Parker RM, Nurss JR. Relationship of functional health literacy to patients' knowledge of their chronic disease: a study of patients with hypertension and diabetes. Archives of internal medicine. 1998;158:166-72.

https://doi.org/10.1001/archinte.158.2.166

10. Rawal I, Ghosh S, Hameed SS, Shivashankar R, Ajay VS, Patel SA, et al. Association between poor oral health and diabetes among Indian adult population: potential for integration with NCDs. BMC oral health. 2019;19:191.(1-0)

https://doi.org/10.1186/s12903-019-0884-4

11. Wehmeyer MM, Corwin CL, Guthmiller JM, Lee JY. The impact of oral health literacy on periodontal health status. J Public Health
Association of oral health literacy level and periodontal health status

Dentistry. 2014;74:80-7

https://doi.org/10.1111/j.1752-7325.2012.00375.x

12. Dental NIo, Craniofacial Research NIoH, US Public Health Service, US Department of Health, Services H. The invisible barrier: literacy and its relationship with oral health. A report of a workgroup sponsored by the National Institute of Dental and Craniofacial Research, National Institute of Health, US Public Health Service, Department of Health and Human Services. J Public Health Dent. 2005;65:174-82. https://doi.org/10.1111/j.1752-7325.2005.tb02808.x

13. da Costa Dutra L, de Lima LCM, Neves ÉTB, Gomes MC, de Araújo LJS, Forte FDS, et al. Adolescents with worse levels of oral health literacy have more cavitated carious lesions. PloS one. 2019;14. https://doi.org/10.1371/journal.pone.0225176

14. Saini R, Marawar P, Shete S, Saini S. Periodontitis, a true infection. J Global infectious diseases. 2009;1:149-50.

https://doi.org/10.4103/0974-777X.56251

15. Jones M, Lee JY, Rozier RG. Oral health literacy among adult patients seeking dental care. J Am Dent Assoc. 2007;138:1199-208. https://doi.org/10.14219/jada.archive.2007.0344

16. Vann Jr W, Lee JY, Baker D, Divaris K. Oral health literacy among female caregivers: impact on oral health outcomes in early childhood. J Dent Res. 2010;89:1395-400.

https://doi.org/10.1177/0022034510379601

17. Cunha-Cruz J, Hujoel P, Kressin N. Oral health-related quality of life of periodontal patients. J periodontal Res. 2007;42:169-76. https://doi.org/10.1111/j.1600-0765.2006.00930.x

18. Ng SK, Leung WK. Oral health-related quality of life and periodontal status. Community Dentistry Oral Epidemiology. 2006;34:114-22. https://doi.org/10.1111/j.1600-0528.2006.00267.x

19. Dickson-Swift V, Kenny A, Farmer J, Gussy M, Larkins S. Measuring oral health literacy: a scoping review of existing tools. BMC oral health. 2014;14:148.

https://doi.org/10.1186/1472-6831-14-148

20. Naghibi Sistani MM, Montazeri A, Yazdani R, Murtomaa H. New oral health literacy instrument for public health: development and pilot testing. J Invest Clin Dent. 2014;5:313-21.

https://doi.org/10.1111/jicd.12042

21. Macek MD, Haynes D, Wells W, Bauer-Leffler S, Cotten PA, Parker RM. Measuring conceptual health knowledge in the context of oral health literacy: preliminary results. J Pub Healt Dent. 2010;70: 197-204.

https://doi.org/10.1111/j.1752-7325.2010.00165.x

22. Khan K, Ruby B, Goldblatt RS, Schensul JJ, Reisine S. A pilot study to assess oral health literacy by comparing a word recognition and comprehension tool. BMC Oral Health. 2014;14:135Article ID: 15 .

https://doi.org/10.1186/1472-6831-14-135

23. Wanichsaithong P, Goodwin M, Pretty IA. Development and pilot 


\section{Ahmed W/ Aslam M/ Hassan SI/ Ahmed K/}

Bukhari SF/ Mahi P/ Kaleem A/ Zafar F

study of an oral health literacy tool for older adults. J Investigative and Clinical Dentistry. 2019;10:e12465.

https://doi.org/10.1111/jicd.12465

24. Atchison KA, Gironda MW, Messadi D, Der-Martirosian C. Screening for oral health literacy in an urban dental clinic. J Pub Healt Dent. 2010;70:269-75.

https://doi.org/10.1111/j.1752-7325.2010.00181.x

25. Roter DL. Oral literacy demand of health care communication: challenges and solutions. Nursing outlook. 2011;59:79-84. https://doi.org/10.1016/j.outlook.2010.11.005

26. Khan AA, Ijaz S, Ayma S, Qureshi A, Padhiar I, Sufia S, Oral health in Pakistan; A situation analysis. Dev Dent. 2004;5:35-44

27. Abdullah M, Zakar R. Health Literacy in South Asia: Clarifying the Connections between Health Literacy and Wellbeing in Pakistan. Health. 2019;34:575-89.

28. Ahmed W, Shaikh ZN, Soomro JA, Qazi HA, Soomro AK. Assessment of health literacy in adult population of Karachi: a
Association of oral health literacy level and periodontal health status

preliminary investigation for concept-based evidence. Int J Health Promotion Edu. 2018;56:95-104.

https://doi.org/10.1080/14635240.2017.1421866

29. Ahmed W, Shah S, Khayyam U, Sheikh T, Anwer N. Measuring Oral health literacy in dental patients: Contribution towards preventive dentistry in Pakistan. J Pak Dent Assoc. 2017;26:176-80. https://doi.org/10.25301/JPDA.264.176

30. Sistani MMN, Virtanen JI, Yazdani R, Murtomaa H. Association of oral health behavior and the use of dental services with oral health literacy among adults in Tehran, Iran. Eur J Dent. 2017;11:162. https://doi.org/10.4103/ejd.ejd_332_16

31. P. R. GeethaPriya SA, D. Kandaswamy, M. S. Muthu \& Shyam S. . Effectiveness of different modes of school dental health education on the oral health status of children - an interventional study with 2-year follow-up, Int J Health Promotion Edu, 58:1, 13-27. https://doi.org/10.1080/14635240.2019.1658536 